\title{
Improving Energy Efficiency of a Refrigeration System with a Rankine Cycle and an Expander
}

\author{
Alison Subiantoro, TUM CREATE (Technical University of Munich - Campus for Research Excellence And \\ Technological Enterprise)
}

\begin{abstract}
A method to increase energy efficiency of a vapor compression refrigeration system by using a Rankine cycle and an expander is studied. The systems studied include the R134a and the transcritical $\mathrm{CO}_{2}$ cycles with a $5 \mathrm{~kW}$ capacity. The working fluids of the Rankine cycle are R134a, propane and R123. The available heat input power is $1-5 \mathrm{~kW}$. The results show that in the $\mathrm{R} 134 \mathrm{a}$ and $\mathrm{CO}_{2}$ systems, $18-40 \%$ and $30-67 \%$ improvements of Coefficient of Performance (COP), respectively, can be achieved. The method is particularly attractive if there is abundant waste/free heat to be utilized.
\end{abstract}

Keywords - vapor compression, energy efficiency, expander Rankine cycle

\section{INTRODUCTION}

Air conditioning and refrigeration are integral parts of the modern life. Among all the alternative systems, the vapor compression (VC) refrigeration system is the most popular due mainly to its simplicity and compactness. It consists of only four (4) main components, i.e. compressor, condenser, expansion device and evaporator. Heat is absorbed from the cooled room/cabin by the evaporator while heat is rejected to the ambient by the condenser. Power is consumed mainly to operate the compressor. Refrigerant flows through the components continuously while undergoing thermodynamic processes. The schematic diagram of a conventional VC system is shown in Fig. 1.

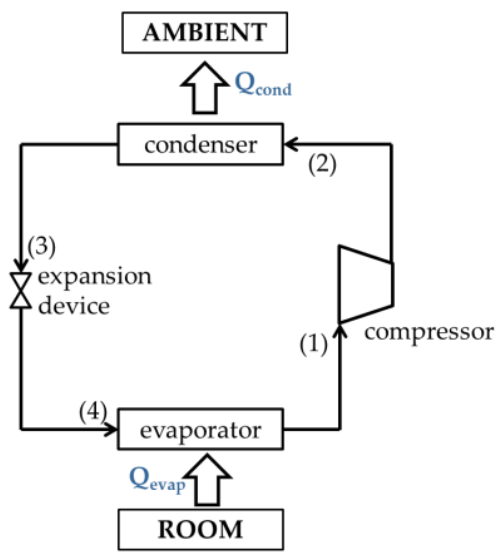

Fig. 1. Schematic of a conventional vapor compression (VC) system.

Unfortunately, air conditioning and refrigeration systems are energy intensive. They account for $40-60 \%$ of the total electricity use of buildings globally [1]. Due to the environmental and economic impacts of electricity power generation, which is mainly done by burning of fossil fuels, it is important to reduce energy consumption of air conditioning and refrigeration systems. Among the methods proposed in the recent years, recovering the usually wasted expansion power at the expansion device using an expander is very attractive. When applied to conventional R22 and R134a systems, the COP (coefficient of performance) has been reported to increase by up to $15 \%$ [2] and $12 \%$ [3], respectively. When applied to a transcritical $\mathrm{CO}_{2}$ system, where the pressure difference between the suction and discharge lines is very high (in the range of $70 \mathrm{bar}$ ), the COP can increase by more than $50 \%$ [4]. Economically, expanders have been shown to be attractive, particularly for regions with high thermal load like in the tropics [5].

Another attractive method that is recently proposed to reduce energy consumption of air conditioning and refrigeration systems is to use waste heat to supply power for the compressor with a Rankine cycle (RC) [6]. A schematic diagram of a RC system is shown in Fig. 2. It consists of four (4) main components, i.e. pump, vaporizer, expander and condense. Heat is absorbed by the vaporizer and rejected to the ambient by the condenser. Useful power is produced by the expander. Selection of the appropriate working fluid is important to optimize the performance of such a system [7-9]. In these reported works, usually the capacity of the VC system is designed according to the power that can be produced by the RC from the available waste/free heat. Therefore, the compressor is wholly powered by the RC. However, even if the RC only partially powered the compressor, the energy efficiency of the VC refrigeration system was still improved.

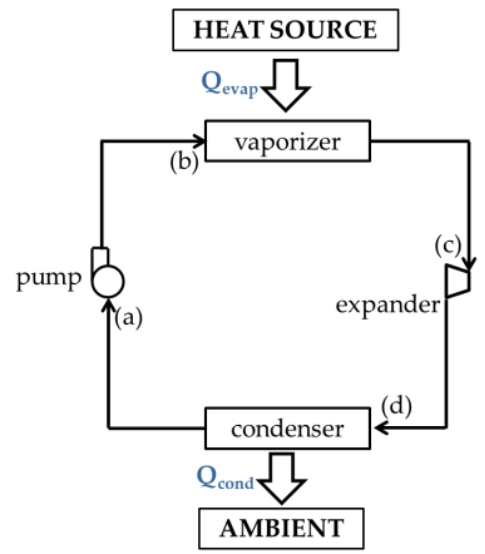

Fig. 2. Schematic of a Rankine cycle (RC) system

The two methods above, i.e. 1) the recovery of expansion work with an expander and 2) the use free heat with a Rankine 
cycle, are not exclusive to each other and can be combined. However, to the author's knowledge, no such attempt has been reported before. It is the purpose of this paper to study such a combined method to improve energy efficiency of a VC refrigeration system. Two different types of VC systems were studied, namely the conventional R134a and the transcritical $\mathrm{CO}_{2}$ systems. The cooling capacity of the $\mathrm{VC}$ refrigeration system was $5 \mathrm{~kW}$. The benchmark working fluid of the RC was R134a. The use of propane (R290) and R123 were also considered for the working fluid of the RC. The available heat input was between 1-5 kW. The schematic diagram of the proposed system is shown in Fig. 3.

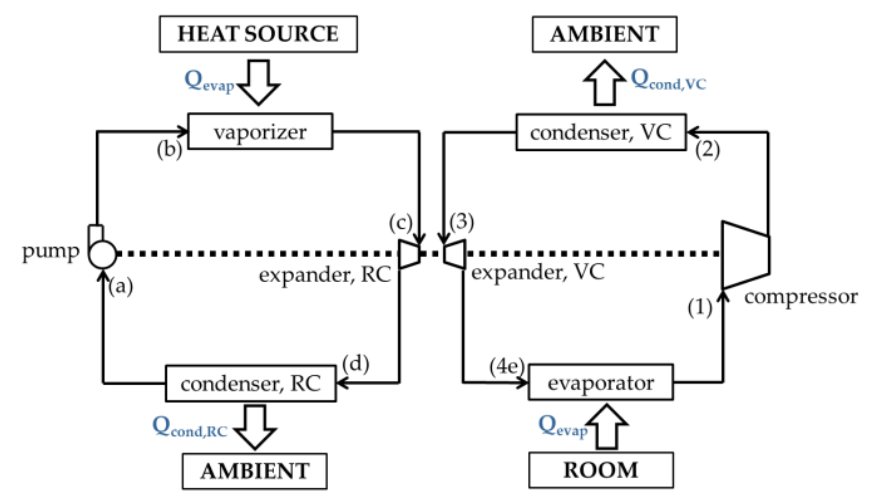

Fig. 3. Schematic diagram of the proposed RC-expander VC system.

As shown in Fig. 3, the proposed system comprises two separate cycles: VC and RC cycles. The VC cycle removes heat from the room while the $\mathrm{RC}$ system converts heat from the heat source to useful energy to help reducing the load of the compressor of VC. To avoid confusion, the working fluid of the VC system will be called "refrigerant" while that of the RC system will be called "working fluid" in this paper.

The working processes of the VC are:

- 1-2: compression of refrigerant by compressor to higher pressure and temperature, power is consumed to operate the compressor during the process;

- 2-3: heat rejection by condenser to the ambient, refrigerant condenses from vapor to vapor-liquid mixture during the process;

- 3-4e: expansion of refrigerant by expander to lower pressure and temperature, power is produced by the expander during the process to lessen the compressor load;

- 4e-1: heat absorption by evaporator from the room, refrigerant evaporates from liquid or vapor-liquid mixture to vapor during the process.

If the $\mathrm{VC}$ system is of the transcritical $\mathrm{CO}_{2}$ cycle, heat rejection to the ambient is done by a gas cooler, not by a condenser, because the process is in the supercritical regime.

The working processes of the RC are:

- a-b: pumping of working fluid by pump to higher pressure, power is consumed to operate the pump during the process;
- b-c: heat absorption by vaporizer from the heat source, working fluid vaporizes from liquid or vapor-liquid mixture to vapor during the process;

- c-d: expansion of working fluid by expander to lower pressure and temperature, power is produced by the expander during the process to lessen the compressor load;

- d-a: heat rejection by condenser to the ambient, working fluid condenses from vapor to liquid or vapor-liquid mixture during the process.

Powers produced by the expanders of $\mathrm{VC}$ and $\mathrm{RC}$ are used to power the pump of $\mathrm{RC}$ and to lessen the load of the compressor of VC.

\section{METHODOLOGY}

To study the system, a computer model was created in MATLAB programming language. Thermo-physical properties of the refrigerants and working fluids were obtained from the REFPROP database [10].

The following assumptions were adopted for the VC model:

- State of refrigerant at the inlet of the compressor was saturated vapor;

- Isentropic compression in the compressor;

- Total efficiency of the compressor was 70\%;

- Isenthalpic expansion in the benchmark expansion valve;

- Isentropic expansion process in the expander of $\mathrm{VC}$, resulting in a cooling capacity increase as compared to the expansion through a valve;

- Total efficiency of the expander was $70 \%$;

- Benchmark evaporating temperature was $5^{\circ} \mathrm{C}$;

- Negligible pressure losses;

- Benchmark cooling capacity was $5 \mathrm{~kW}$;

- In the R134a vapor compression cycle:

o State of R134a at the inlet of the expansion device was saturated liquid;

○ Benchmark condensing temperature was $50^{\circ} \mathrm{C}$;

- In the transcritical $\mathrm{CO}_{2}$ vapor compression cycle:

○ State of $\mathrm{CO}_{2}$ at the inlet of the expansion device was supercritical gas at $35^{\circ} \mathrm{C}$;

- Benchmark gas cooler pressure was $10 \mathrm{MPa}$ (100 bar).

The following assumptions were adopted for the RC model:

- State of working fluid at the inlet of the expander was saturated gas;

- Isentropic expansion process in the expander of RC;

- Total efficiency of expander of RC was 70\%;

- State of working fluid at the inlet of the pump was saturated liquid;

- Isentropic pumping process in the pump;

- Total efficiency of pump was $70 \%$;

- Vaporizing temperature was $80^{\circ} \mathrm{C}$;

- Negligible pressure losses;

- Benchmark external heat input was $1 \mathrm{~kW}$;

- Condensing temperature of RC was:

$\circ$ Equal to the condensing temperature of $\mathrm{VC}$ if for the R134a VC system;

o Constant of $50^{\circ} \mathrm{C}$ for the transcritical $\mathrm{CO}_{2} \mathrm{VC}$ system. 
Temperature-entropy diagrams of the benchmark R134a VC cycle, the R134a VC cycle with an expander and the R134a $\mathrm{RC}$ are shown in Fig. 4. Locations of points (a) and (b) are very close to each other but are not the same. If the VC system is of the transcritical $\mathrm{CO}_{2}$ cycle, the $\mathrm{VC}$ cycle will be at higher operating pressure conditions (in the range of 40 to 120 bar) as compared to the R134a system (in the range of 3 to 20 bar).

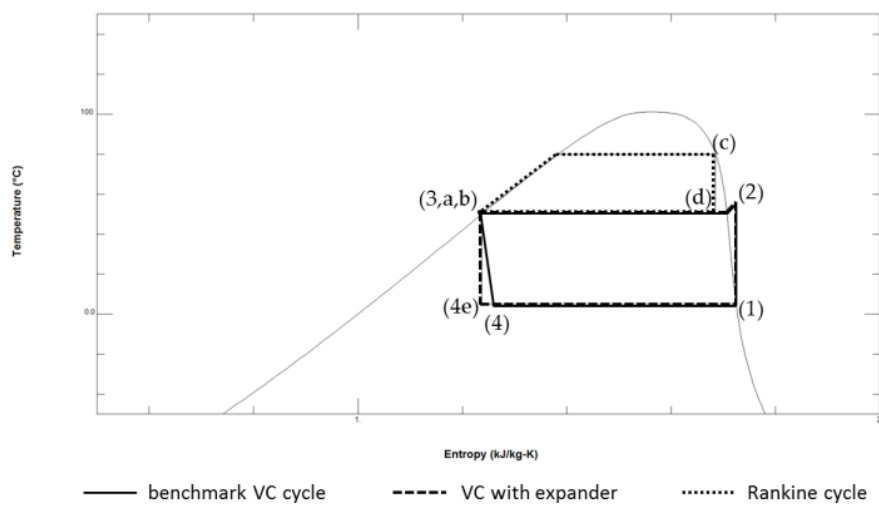

Fig. 4. Temperature-entropy diagrams of the benchmark and the proposed cycles with R134a VC system.

The compressor power requirement was calculated according to (1), where $W$ is work done (W), $\dot{m}$ is mass flow rate $(\mathrm{kg} / \mathrm{s}), h$ is specific enthalpy $(\mathrm{J} / \mathrm{kg})$ and $\eta$ is efficiency (-).

$$
W_{\text {comp }}=\dot{m}_{V C}\left(h_{2}-h_{1}\right) / \eta_{\text {comp }}
$$

The heat flux rejected by the condenser of $\mathrm{VC}$ was calculated according to (2), where $Q$ is heat flux (W). If the $\mathrm{VC}$ system is of the transcritical $\mathrm{CO}_{2}$ cycle, heat is rejected by a gas cooler, not a condenser, but the heat flux is still calculated according to (2).

$$
Q_{\text {cond,VC }}=\dot{m}_{V C}\left(h_{2}-h_{3}\right)
$$

The power produced by the expander of $\mathrm{VC}$ was calculated according to (3).

$$
W_{\text {exp,VC }}=\dot{m}_{V C}\left(h_{3}-h_{4 e}\right) \times \eta_{\text {exp } y C}
$$

The cooling capacity of the evaporator was calculated according to (4). In the simulation, the equation was used to calculate the mass flow rate of refrigerant of $\mathrm{VC}$ because cooling capacity and states of refrigerants at the inlet and outlet of evaporator were known.

$$
Q_{\text {evap }}=\dot{m}_{V C}\left(h_{1}-h_{4 e}\right)
$$

The heat flux at the vaporizer was calculated according to (5). This was also equal to the input heat flux from the external heat source. In the simulation, the equation was used to calculate the mass flow rate of the working fluid of $\mathrm{RC}$ because the input heat flux and states of refrigerants at the inlet and outlet of vaporizer were known.

$$
Q_{v a p}=\dot{m}_{R C}\left(h_{c}-h_{b}\right)
$$

The power produced by the expander of $\mathrm{RC}$ was calculated according to (6).

$$
W_{\text {exp }, R C}=\dot{m}_{R C}\left(h_{c}-h_{d}\right) \times \eta_{\text {expRC }}
$$

The heat flux of the condenser of $\mathrm{RC}$ was calculated according to (7).

$$
Q_{\text {cond }, R C}=\dot{m}_{R C}\left(h_{d}-h_{a}\right)
$$

The pump power requirement of RC was calculated according to (8).

$$
W_{\text {pump }}=\dot{m}_{R C}\left(h_{b}-h_{a}\right) / \eta_{\text {pump }}
$$

The benchmark COP (Coefficient of Performance) was calculated according to (9), assuming that the expansion process in the expansion valve was isenthalpic.

$$
C O P_{\text {benchmark }}=\frac{\left(h_{1}-h_{3}\right)}{\left(h_{2}-h_{1}\right) / \eta_{\text {comp }}}
$$

The COP (Coefficient of Performance) of the system with an expander was calculated according to (10). The cooling capacity is calculated assuming that the expansion process in the expander is isentropic.

$$
C O P_{w / \exp }=\frac{Q_{\text {evap }}}{W_{\text {comp }, V C}-W_{\text {exp } V C}}
$$

The COP (Coefficient of Performance) of the system with RC but without expander was calculated according to (11). The cooling capacity is calculated assuming that the expansion in the valve is isenthalpic.

$$
C O P_{w / R C}=\frac{\dot{m}_{V C}\left(h_{1}-h_{3}\right)}{W_{c o m p, V C}-W_{\text {expRC }}+W_{\text {pump }, R C}}
$$

The COP (Coefficient of Performance) of the system with $\mathrm{RC}$ and with expander was calculated according to (12).

$$
C O P_{w / R C \& \exp }=\frac{Q_{\text {evap }}}{W_{\text {comp }, V C}-W_{\text {exp } y C}-W_{\text {exp }, R C}+W_{\text {pump }, R C}}
$$

It is useful to note that the definitions of COPs in (11) and (12) are given according to the usual method of quantifying the efficiency of a VC refrigeration system. A different definition of COP, which calculates the ratio between the cooling capacity and the input heat flux, is sometimes used to quantify the efficiency of an integrated VC-RC system [6-9]. This definition is useful when the size of the VC system is designed according to the amount of input heat available. In such a system, the compressor is powered wholly by the 
power produced by the RC. However, in this study, the reverse is true where the capacity of the VC system is set and the available heat input is used to improve the VC system. Therefore, this latter definition of COP will not be considered in this paper.

\section{RESUlTS AND DisCUSSIONS}

\section{A. R134a vapor compression system}

The results of the study are discussed in the following section. The discussion is started with the R134a VC system. Comparisons of COP values of the benchmark VC system, VC system with an expander, VC system with RC but without expander and VC system with RC and expander are shown in Fig. 5. The variations of COP values with external input heat flux, evaporating and condensing temperatures are also shown in the figure.
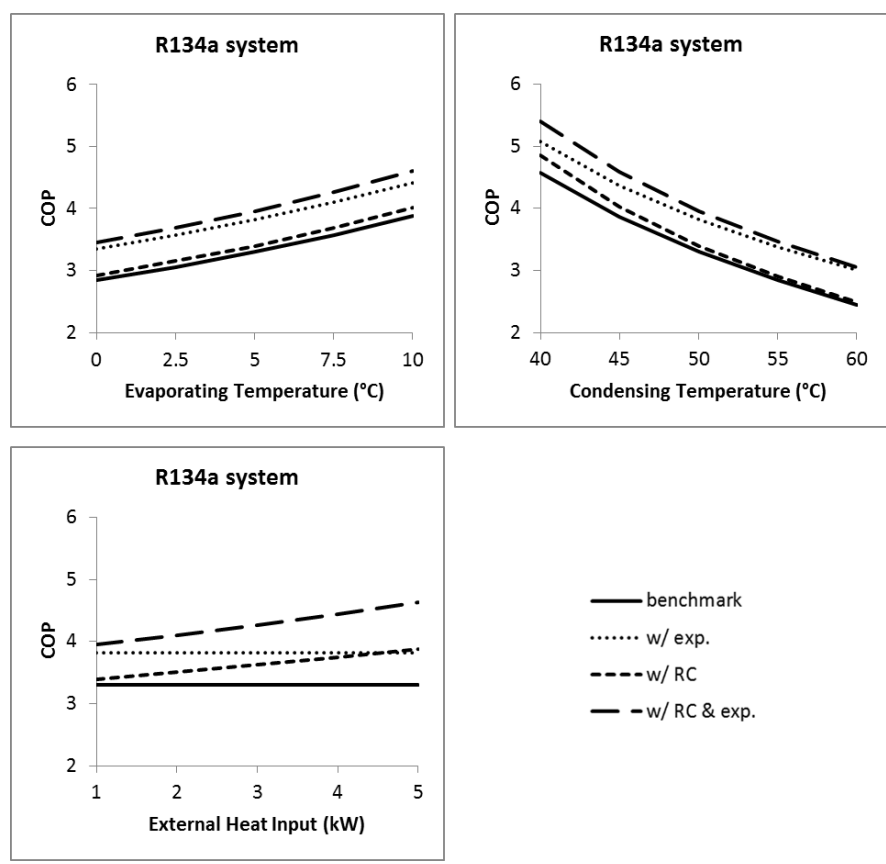

Fig. 5. Comparison of COP values at various evaporating and condensing temperatures of: 1) the benchmark VC system ("benchmark"), 2) VC with an expander system ("w/ exp."), 3) VC with RC system ("w/ RC") and 4) VC with RC and expander system ("w/ RC \& exp.). The refrigerant of VC and working fluid of RC are both R134a.

It can be seen from Fig. 5 that with a higher evaporating temperature or a lower condensing temperature, the COPs of all the systems were higher. The main reason is the corresponding smaller compressor load. For the expander of $\mathrm{VC}$, a higher evaporating temperature or a lower condensing temperature reduced its power production due to the smaller pressure drop available. For the RC system, its power production was independent of the evaporating temperature but was affected by the condensing temperature because its condensing temperature was always equal to that of the $\mathrm{VC}$ in this study. Therefore, with a lower condensing temperature, the $\mathrm{RC}$ produced more power as there was a higher pressure drop across the expander of RC.
Individually, expander of $\mathrm{VC}$ improved the COP more significantly (between 11-23\%) than RC (between 2-6\%) when only $1 \mathrm{~kW}$ of heat is available. The expander's contribution was even higher at higher condensing temperatures. At such conditions, as explained above, the expander of $\mathrm{VC}$ produced more power while the $\mathrm{RC}$ produced less power. At higher evaporating temperatures, the compressor required less power, the expander produced less power while the RC was unaffected. Therefore, the contribution of $\mathrm{RC}$ to the COP increase was higher at a high evaporating temperature.

When the available heat was $5 \mathrm{~kW}$, the contribution of $\mathrm{RC}$ was comparable to that of the expander and the total COP improvement was up to $40 \%$. Comparably, if only an expander was installed, only around 15\% COP improvement was achieved. Moreover, if only $\mathrm{RC}$ was installed, the COP increase was $18 \%$.

COP improvements and performance comparison of three different RC working fluids, i.e. R134a, propane and R123, are shown in Fig. 6. At the benchmark conditions, COP improvement is around 20\%. Between 18-25\% COP improvement was achievable across the various evaporating and condensing temperature conditions studied here, when there was $1 \mathrm{~kW}$ of heat available. The highest COP improvement was achieved when the condensing temperature was $60^{\circ} \mathrm{C}$. The COP improvement was higher at a lower evaporating temperature or a higher condensing temperature due to the higher power produced by the expander of $\mathrm{VC}$. In terms of the total contribution to the COP improvement, the expander of $\mathrm{VC}$ and the RC contributed between $46-93 \%$ and $7-54 \%$, respectively, when only $1 \mathrm{~kW}$ was available.
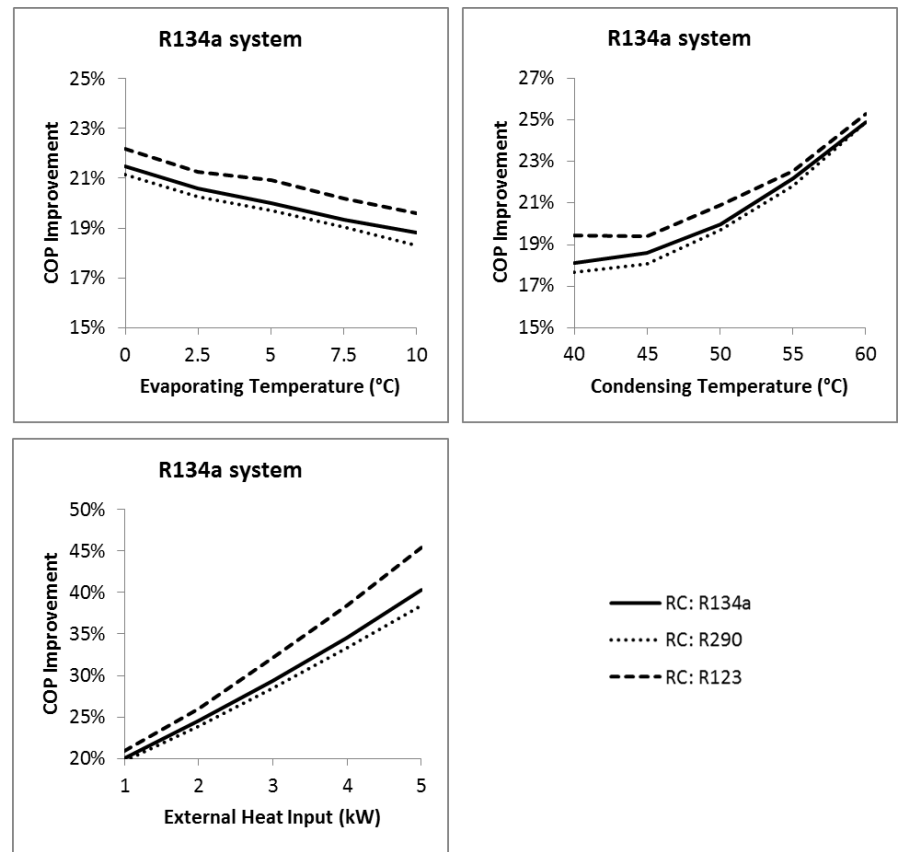

Fig. 6. Comparison of COP improvements of the proposed system (R134a is the refrigerant of VC) with R134a, R290 (propane) and R123 as the working fluid of RC. 
Fig. 6 also shows that R123 was the most preferred working fluid while propane (R290) was the least preferred. R123 outperformed the other working fluids in all operating conditions tested in the study, particularly at lower condensing temperature and high external heat input conditions. At the benchmark operating conditions, R123 improved the COP of the VC system by $21 \%$ while the other working fluids were only able to improve by $20 \%$. When the available heat input was $5 \mathrm{~kW}$, up to $45 \%$ in COP improvement was achievable by R123 while the other working fluids can only achieve around 40\% COP improvement.

\section{B. Transcritical $\mathrm{CO}_{2}$ vapor compression system}

Performances of the benchmark transcritical $\mathrm{CO}_{2} \mathrm{VC}$ system, VC system with an expander, VC system with RC but without expander and VC system with RC and expander were compared in Fig. 7. Like that in the R134a system, COPs increased with evaporating temperature because of the smaller compressor load. However, the trend with varying gas cooler pressure of the transcritical $\mathrm{CO}_{2} \mathrm{VC}$ system was different from that of varying the condenser temperature of the R134a system. An optimum gas cooler pressure was observed, which is typical for transcritical $\mathrm{CO}_{2}$ systems. The optimum gas cooler pressure was around 90 bar in the systems studied.
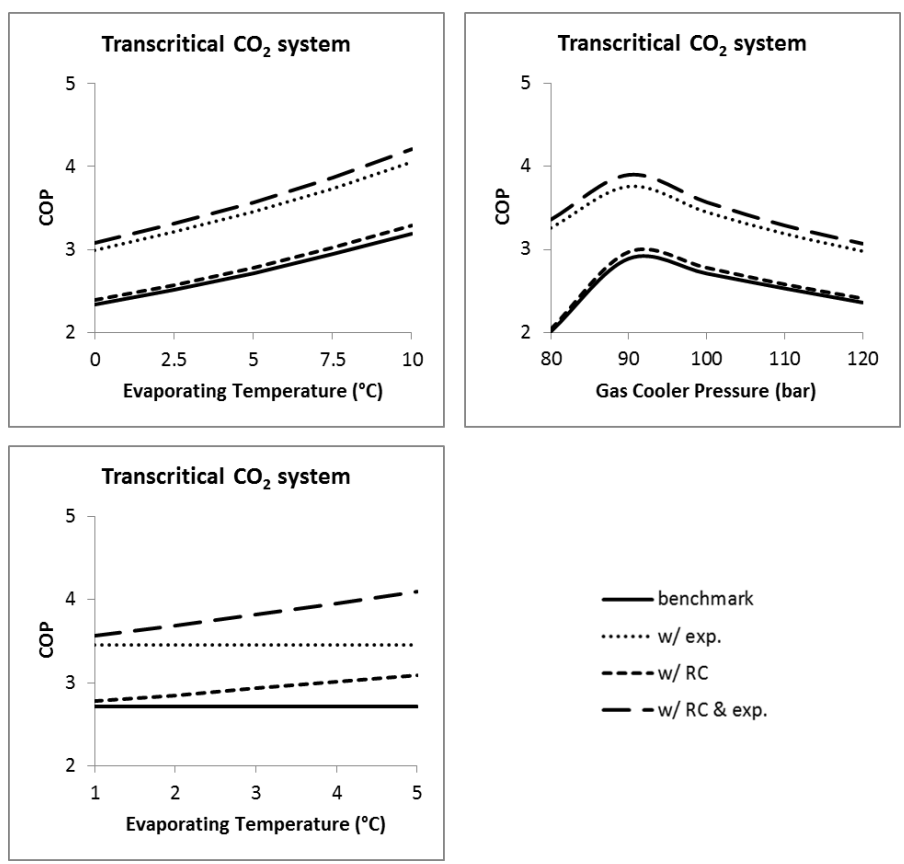

Fig. 7. Comparison of $\mathrm{COP}$ values at various evaporating and condensing temperatures of: 1) the benchmark VC system ("benchmark"), 2) VC with an expander system ("w/ exp."), 3) VC with RC system ("w/ RC") and 4) VC with $\mathrm{RC}$ and expander system ("w/ RC \& exp.). The refrigerant of $\mathrm{VC}$ is $\mathrm{CO}_{2}$ while the working fluid of RC is R134a.

Another observation from Fig. 7 is that the contribution of expander to the overall COP increase was even more significant than the RC as compared to that in the R134a system discussed in the previous section. Individually, expander improved the $\mathrm{COP}$ of the $\mathrm{CO}_{2} \mathrm{VC}$ system by between 26-62\% while RC improved the COP by only between $1-3 \%$ when $1 \mathrm{~kW}$ of heat was available. When there was $5 \mathrm{~kW}$ of heat available, RC alone could increase the COP by $14 \%$ and the contribution of $\mathrm{RC}$ to the overall COP increase was still behind (only around half) that of the expander. Comparably, if only an expander was used, $27 \%$ of COP improvement was achieved. This phenomenon occurred because expanders are most effective in high pressure operating conditions like in a transcritical $\mathrm{CO}_{2} \mathrm{VC}$ system where the expansion pressure drop is large. Moreover, a transcritical $\mathrm{CO}_{2}$ system usually has a higher compressor load as compared to an R134a system. Meanwhile, the amount of power produced by the $\mathrm{RC}$ was independent of the type of refrigerant in the $\mathrm{VC}$ system. Therefore, the final contribution of RC to the COP increase of the $\mathrm{CO}_{2} \mathrm{VC}$ system was less as compared to that to an R134a system. Nonetheless, the combined method of RC and expander could improve the COP by more than $50 \%$ if there was $5 \mathrm{~kW}$ of heat available to be used by RC. Therefore, as in the case of an R134a system, RC is attractive if there is abundant heat available to be used.

COP improvements and performance comparison of various different RC working fluids are shown in Fig. 8. In general, between 30-67\% COP improvement was achievable with the proposed combined method across the various evaporating temperature and gas cooler pressure conditions when $1 \mathrm{~kW}$ of heat was available. At the benchmark conditions, the COP improvement is around $32 \%$. The highest COP improvement was at gas cooler pressure of 80 bar. This large improvement was because the expander is very effective for a transcritical $\mathrm{CO}_{2}$ system and the benchmark COP value was low at 2.0.

Among the three working fluids tested, R123 was again the most preferred. In the benchmark system, it can improve the COP by $33 \%$ while the other fluids can only improve by $32 \%$. When there was $5 \mathrm{~kW}$ of heat available to be used, R123 could achieve COP improvement of more than $56 \%$.
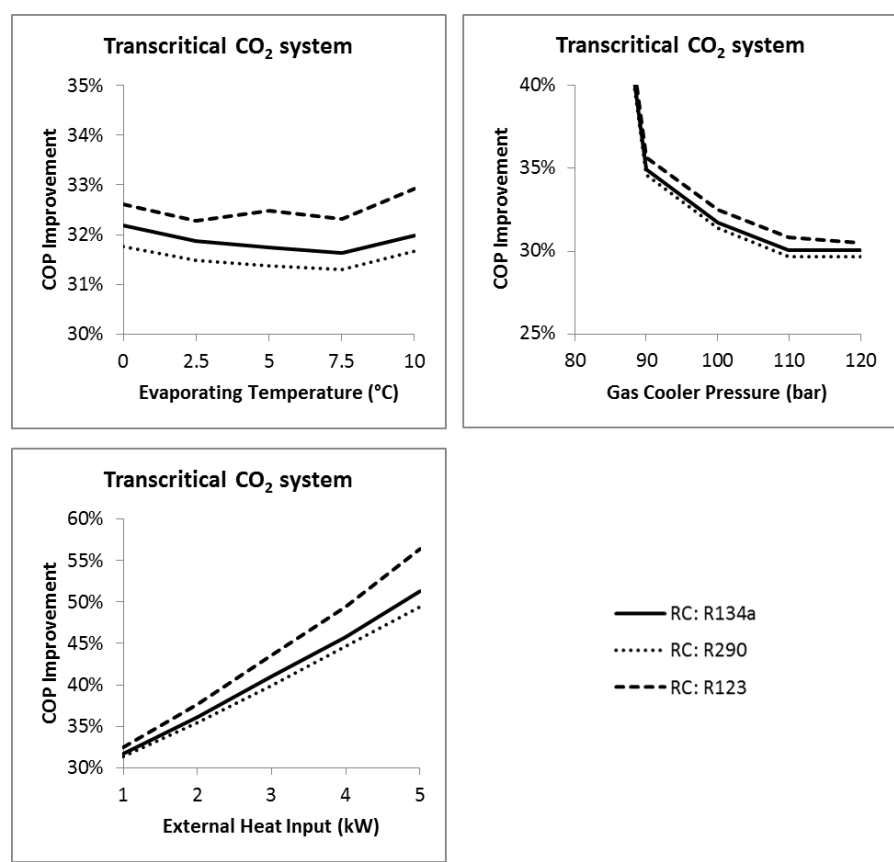

Fig. 8. Comparison of COP improvements of the proposed system $\left(\mathrm{CO}_{2}\right.$ is the refrigerant of VC) with R134a, R290 (propane) and R123 as the working fluid of RC. 
From the results obtained, it can be seen that if there is abundant free heat to be utilized, it is attractive to install the proposed combined RC-expander system. Otherwise, considering the additional cost and size involved with the installation of a RC system, it is more practical to install an expander alone. The additional size and weight of the RC system will also limit its implementation to stationary applications.

\section{CONCLUSIONS}

A combined method to increase energy efficiency of vapor compression (VC) refrigeration systems was studied. The method integrated the recovery of expansion work using an expander and the use of waste/free heat with a Rankine cycle (RC). Two different types of VC systems were studied, i.e. the conventional $\mathrm{R} 134 \mathrm{a}$ and the transcritical $\mathrm{CO}_{2}$ systems. The cooling capacity of the VC refrigeration system was $5 \mathrm{~kW}$. The benchmark working fluid of the RC was R134a. The use of propane (R290) and R123 were also considered for the RC. The available heat input was between $1-5 \mathrm{~kW}$. The study was carried out by computational simulation of the system.

The study with an R134a VC system found the followings:

- At the benchmark conditions, COP improvement is around $20 \%$.

- With a higher evaporating temperature or a lower condensing temperature, the COPs were higher because of the corresponding smaller compressor load.

- Between 18-25\% total COP improvement was achievable across various evaporating and condensing temperature conditions studied here when there was $1 \mathrm{~kW}$ of heat available.

- Individually, expander improved the COP more significantly (between 11-23\%) than RC (between 2$6 \%$ ) when only $1 \mathrm{~kW}$ of heat was available.

- In terms of the total contribution to the COP improvement, the contributions of the expander and the RC were between and 46-93\% and 7-54\%, respectively, when only $1 \mathrm{~kW}$ was available. The expander's contribution was notably higher than $\mathrm{RC}$ at higher condensing temperatures. At such conditions, the expander produced more power while the $\mathrm{RC}$ produced less power.

- When the available heat input was around $5 \mathrm{~kW}$, the contribution of RC was similar to that of the expander and the total COP improvement was up to $40 \%$. Comparably, if only an expander was installed, COP increase was $15 \%$. If only RC was installed, the COP increase was $18 \%$.

- R123 was the most preferred working fluid while propane (R290) was the least preferred. At the benchmark operating conditions, R123 improved the COP of the VC system by $21 \%$. When the available heat input was $5 \mathrm{~kW}$, up to $45 \%$ in COP improvement was achievable by R123.

The study with a transcritical $\mathrm{CO}_{2} \mathrm{VC}$ system found the followings:
- At the benchmark conditions, COP improvement is around $32 \%$.

- COP increased with evaporating temperature because of the smaller compressor load, similar to that found in an R134a system.

- An optimum gas cooler pressure was observed. The optimum gas cooler pressure was around 90 bar in the systems studied here.

- Between 30-67\% COP improvement was achievable with the proposed combined method across various evaporating temperature and gas cooler pressure conditions when $1 \mathrm{~kW}$ of heat was available.

- Expander's contribution to the COP increase was more significant than that of the RC. The difference is even larger than in the R134a VC system. Individually, expander improved the $\mathrm{COP}$ of the $\mathrm{CO}_{2} \mathrm{VC}$ system by between $26-62 \%$ while RC improved the COP by only between $1-3 \%$ when $1 \mathrm{~kW}$ of heat was available.

- The combined method of RC and expander could improve the COP by more than $50 \%$ if there was $5 \mathrm{~kW}$ of heat available to be used by RC. Comparably, RC alone could increase the COP by $14 \%$ when there was $5 \mathrm{~kW}$ of heat available. If only an expander was used, $27 \%$ of COP improvement was achieved.

- Among the alternative working fluids studied, R123 was the most preferred. In the benchmark system, it can improve the COP by $33 \%$. It could achieve COP improvement of more than $56 \%$ when there was $5 \mathrm{~kW}$ of heat available to use.

To conclude, the proposed combined RC-expander method is able to improve energy efficiency of $\mathrm{VC}$ systems. It is particularly attractive if there is abundant waste/free heat to be utilized. In situations where there is limited free heat available, considering the additional cost and size involved with the installation of a RC system, it is more practical to install an expander alone.

\section{ACKNOWLEDGMENT}

This work was financially supported by the Singapore National Research Foundation under its Campus for Research Excellence and Technological Enterprise (CREATE) program.

\section{REFERENCES}

[1] L. Perez-Lombard, J. Ortiz and C. Pout, "A review on buildings energy consumption information," Energ. Buildings, vol. 40, no. 3, pp. 394-398, 2008. http://dx.doi.org/10.1016/j.enbuild.2007.03.007

[2] D. M. Robinson, and E. A. Groll, "Efficiencies of transcritical $\mathrm{CO}_{2}$ cycles with and without an expansion turbine," Int. J. Refrig., vol. 21, no. 7, pp. 577-589, November 1998. http://dx.doi.org/10.1016/S01407007(98)00024-3

[3] V. D. A. Goncalves and J. A. R. Parise, "A study on the reduction of throttling losses in automotive air conditioning systems through expansion work recovery," in International Refrigeration and Air Conditioning Conference at Purdue, 2008, pp. 2416:1-8.

[4] M. Fukuta, T. Yanagisawa, S. Nakaya and Y. Ogi, "Performance and characteristics of compressor/expander combination for $\mathrm{CO}_{2}$ cycle," in: 7th IIR Gustav Lorentzen Conference on Natural Working Fluids, 2006, pp. 052:1-8.

[5] A. Subiantoro, K. T. Ooi, "Economic analysis of the application of expanders in medium scale air-conditioners with conventional 
refrigerants, R1234yf and $\mathrm{CO}_{2}$,", Int. J. Refrig., vol. 36, no. 5, pp. 14721482, August 2013. http://dx.doi.org/10.1016/j.ijrefrig.2013.03.010

[6] S. Aphornratana and T. Sriveerakul, "Analysis of a combined Rankinevapour-compression refrigeration cycle," Energ. Convers. Manage., vol 51, no. 12, pp. 2557-2564, December 2010 http://dx.doi.org/10.1016/j.enconman.2010.04.016

[7] Y.-R. Li, X.-Q. Wang, X.-P. Li and J.-N. Wang, "Performance analysis of a novel power/refrigerating combined-system driven by the low-grade waste heat using different refrigerants," Energy, vol. 73, pp. 543-553, August 2014. http://dx.doi.org/10.1016/j.energy.2014.06.054

[8] H. Li, X. Bu, L. Wang, Z. Long and Y. Lian, "Hydrocarbon working fluids for a Rankine cycle powered vapor compression refrigeration system using low-grade thermal energy," Energ. Buildings, vol. 65, pp. 167-172, October 2013. http://dx.doi.org/10.1016/j.enbuild.2013.06.012

[9] X. Bu, L. Wang and $\mathrm{H}$. Li, "Working fluids selection for fishing boats waste heat powered organic Rankine-vapor compression ice maker," Heat Mass Transfer, vol. 50, pp. 1479-1485, October 2014. http://dx.doi.org/10.1007/s00231-014-1350-0

[10] E. W. Lemmon, M. L. Huber and M. O. McLinden, "NIST Standard Reference Database 23: Reference Fluid Thermodynamic and Transport Properties-REFPROP, Version 9.0", National Institute of Standards and Technology, Standard Reference Data Program, Gaithersburg, 2010. 